\title{
Analysis of the Principle and Application of Dynamic Reactive Power Compensation Device
}

\author{
Tianqi Lu ${ }^{1, a}$, Jiye Yang ${ }^{1, b}$, Zezhong Wang ${ }^{2, c}$, Jinyuan Liu ${ }^{1}$, Li Jiang ${ }^{1}$, Meijun \\ $\mathrm{Li}^{1}$, Zhuoran Song ${ }^{1, \mathrm{~d}}$, Qiang $\mathrm{Ma}^{1}$, Zheng $\mathrm{Gu}^{1}$ \\ ${ }^{1}$ State Grid Liaoning Electric Power Company Limited Economic Research Institute, Shenyang, \\ 110015, China
}

\author{
${ }^{2}$ State Grid Beijing Power Supply Company, Beijing,China \\ aemail: lutianqi5558972@sina.com, bemail: yjy-cmy@163.com

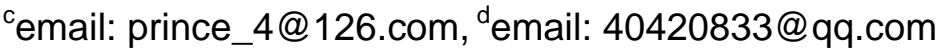

Keywords: Application analysis; Dynamic reactive power compensation; Power Systems

\begin{abstract}
Reasonable reactive power compensation is very important for the transmission and distribution system, the current main dynamic compensation for the TCR SVC, MCR SVC and SVG three ways. In this paper, the working characteristics and basic principles of these three methods are introduced, and the development direction of reactive power compensation and harmonic suppression technology is obtained by analyzing and comparing the performance indexes such as response speed, harmonic characteristic, equipment loss and floor space, And application prospects.
\end{abstract}

\section{Introduction}

With the continuous development of power grids, the importance of control and compensation for reactive power is increasing. If the reactive power reserve will lead to lower grid voltage level, the impact of the reactive power load will make the voltage fluctuations, deterioration of the power supply quality. At present, the reactive power compensation device has been developed by synchronous modulator and shunt capacitor to dynamic reactive power compensation device based on high power power electronic device. Static Var Compensation (hereinafter referred to as SVC), is a fast adjustment of dynamic reactive power device, which typically represents the thyristor control reactor + fixed capacitor (TCR type) and magnetically controlled reactor + fixed Capacitor (MCR type)[1]. SVC has been successfully applied to wind turbine, metallurgical and electrified railway and other impact load on the compensation. This kind of device has obvious advantages in the aspect of adjusting the fastness, the diversity of function, the reliability of operation and the economy of investment and running cost, and has achieved good technical and economic benefits. Rapid development and practical application. Based on the research of SVC technology, Static Var Generation (SVG) has become the latest generation of dynamic reactive power compensation technology with the development and application of high power power electronic devices such as GTO, IGCT and IGBT. It has the advantages of fast response speed, continuous reactive power generation, high harmonic quantity, wide adjustment range, low loss and noise, and plays an increasingly important role in the field of power quality and reactive power compensation research. The following analysis of the typical characteristics of TCR SVC, MCR SVC and SVG can be used to judge the future development trend of reactive power compensation technology and the popularization and application of new technology.

\section{TCR-type SVC dynamic reactive power compensation device}

\section{TCR-type SVC equipment principle.}

TCR (TCR: Thyristor Control Reactor thyristor control reactor) type SVC equipment consists of two parts of TCR and FC, FC to the system to provide a fixed capacitive reactive power and filter 
the high voltage bus on the harmonics; TCR thyristor control reactor Device, by the control system real-time tracking load changes to change the trigger angle of the thyristor to the system to provide real-time variable inductive reactive power. Capacitive reactive and inductive reactive phase, the addition of the two will change the amount of reactive power change, so as to achieve the suppression of voltage fluctuations, improve the power factor and so on. FC is directly connected to the high-voltage bus under a fixed number of fixed filters, the filter impedance curve fixed, can change the load changes in the process of effective harmonics filter to meet the national standard; TCR fast tracking load changes ( Response time is less than 10ms).

\section{TCR-type SVC equipment performance indicators.}

\section{1) Response speed}

TCR-type SVC device dynamic part of the use of thyristor phase-controlled reactor, SVC dynamic part of the response time can be less than $10 \mathrm{~ms}$, and for the smooth adjustment to meet the load dynamic reactive power compensation fast and accurate requirements.

2) Harmonic characteristics

TCR itself is a rectifier load, when the TCR put into work when they produce a large harmonic current. TCR-type SVC equipment through the FC part of the grid with the characteristics of the same harmonic filter to filter the harmonic.

3) Equipment loss

SVC equipment installed directly on the high side, the operating current is small, the statistics, TCR-type SVC equipment, the average loss of equipment compensation capacity of $0.9 \%$ to $1.2 \%$.

4) Adjust the characteristics

TCR-type SVC by adjusting the triggering angle of the thyristor to change the reactive output of the TCR, so you can get a linear smooth reactive output.

5) Land area and environment

As the TCR-type SVC using high-pressure thyristor valve group and the hollow phase-controlled reactor, and FC filter group, so the corresponding area is larger. Will produce a certain amount of electromagnetic radiation.

\section{MCR-type SVC dynamic reactive power compensation device}

\section{MCR-type SVC equipment principle.}

MCR (MCR: Magnetic Control Reactor magnetic valve type control reactor) 60 years by the British GEC company made the first self-saturation reactor type SVC, the late Russian people evolved into a controlled saturation reactor (CSR) Also known as MCR dynamic reactive power compensation device. The principle is that the working windings of the three-phase saturated reactors are connected in parallel to the grid. By changing the excitation current of the DC-controlled windings of the saturable reactor, the saturation characteristics of the core are changed to change the inductance of the working windings to change the absorption The purpose of reactive power.

The main core of the magnetic valve type controllable reactor is split into two halves (ie, core 1 and core 2), the cross-sectional area is A, the cross-sectional area of each half core has a reduced section, and the four turns are $\mathrm{N} / 2$ Symmetrically around the two semi-core pillars (semi-core column on the total number of turns $\mathrm{N}$ ), each half of the core of the upper and lower windings have a tap ratio of $\delta=\mathrm{N} 2 / \mathrm{N}$ of the tap, they are connected between the thyristor KP1 (KP2), the upper and lower windings on the different cores are cross-connected, connected to the grid power supply, and the freewheeling diode is across the crossed ends. In the whole capacity adjustment range, only the small area of the magnetic field saturation, the rest are in the unsaturated linear state, by changing the saturation of the small section of the magnetic circuit to change the capacity of the reactor.

In the power of a power frequency cycle, the thyristor KP1, KP2 turns from the role of full-wave rectifier, the diode plays a role in freewheeling. Changing the firing angle of KP1, KP2 can change the size of the control current, thus changing the saturation of the reactor core to smooth and continuously adjust the capacity of the reactor. 


\section{MCR-type SVC equipment performance indicators.}

\section{1) Response speed}

MCR-type SVC response speed is generally within $100 \sim 200 \mathrm{~ms}$. Controlled Saturation Reactor The magnetic flux in the core has inertia, from no load to rated change, usually above the second. Although some measures can now be taken to improve the response speed of the MCR SVC, it is generally difficult to less than $100 \mathrm{~ms}$.

According to the literature, the response time of the solenoid valve type controllable reactor is determined by the following equation (1). Where $\mathrm{n}$ is the number of frequency cycles required for the capacity of the solenoid-type controllable reactor from no load to the rated value, and $\delta$ is the tap ratio. For example, when the MCR tap ratio is 0.05 , the response speed is about 10 cycles, that is, $200 \mathrm{~ms}$; if the tap ratio is smaller, the response is slower.

$$
n=(1-\delta) / 2 \delta
$$

2) Harmonic characteristics

MCR itself to produce a certain harmonic to be equipped with the filter branch filter itself and the system harmonics. If the reactor part of the input, the reactor and the load generated by the harmonics will appear simultaneously.

3) Equipment loss

In this structure, the edge effect of magnetic saturation is significant. Due to the alternating saturation of the magnetic valve, there is a large transverse magnetic field in the core area near the magnetic valve. In this case, the magnetic reactor is in the magnetic saturation region. Component, thus increasing the additional losses of the reactor core and winding, typically about $1 \%$ to $2.0 \%$.

At present, MCR through continuous technological innovation, loss also declined, but never lower than the core reactor rated loss[2]. Core-type reactor of the national standard, according to its different capacity, the rated loss is generally between 1.2 to $2 \%$. Therefore, the actual operation, less than 50,000 MVar MCR-type dynamic compensation loss will not be less than $1.8 \%$

4) Adjust the characteristics

Saturated reactors are non-linear components, so that the current of the working winding can not effectively follow the control winding (field winding) current changes, that is, compensation of reactive power over and under- In order to suppress the over-compensation phenomenon, the design of the control current is limited to the core fully saturated current of 0.85 or less, that is, MCR reactive control range of $0 \sim 85 \%$, rather than $0 \sim 100 \%$.

\section{5) Area}

Because the MCR does not use the thyristor valve group as well as the hollow phase control reactor like the TCR, but uses the thyristor to control the partial saturation reactor, therefore, is smaller than the TCR area. However, due to the inherent characteristics of the core-type saturation reactor, so the actual operation will produce a certain vibration and operating noise.

\section{SVG dynamic reactive power compensation device}

\section{SVG equipment principle.}

Due to the rapid development of power electronics technology, the use of high-power turn-off thyristor (GTO) devices instead of ordinary thyristors constitute the reactive power compensator has begun to enter the practical stage. This device is called static var generator (Static Var Generator, referred to as SVG). SVG can be divided into voltage type and current type two types, the DC side respectively using capacitance and inductance as energy storage components. In fact, due to operational efficiency reasons, so far put into practical SVG mostly use voltage bridge circuit, as shown in Figure 1, its structure is simple, low energy loss, low cost and easy to control, so SVG is often specifically used to change Phase voltage bridge converter circuit as a dynamic reactive power compensation device. 


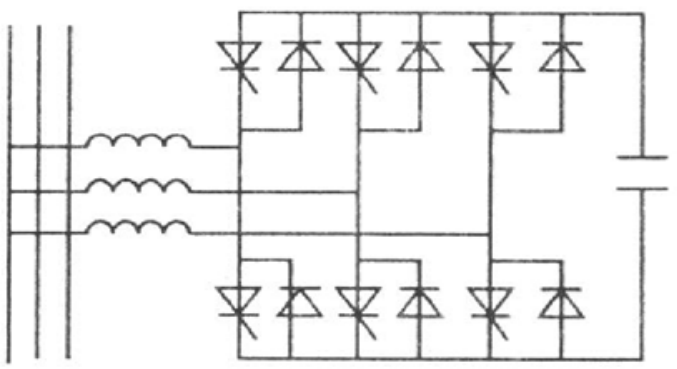

Figure.1. SVG circuit structure

\section{SVG equipment performance indicators.}

Essentially, SVG can be equivalent to a capacitor or reactor that can be continuously adjusted in size.

1) response time

SVG response time: $\leqslant 5 \mathrm{~ms}$.

SVG can be completed within a very short period of time from the rated capacitive reactive power to the rated inductive reactive power conversion, fully capable of compensation for the impact load.

2) Harmonic characteristics

As the SVC dynamic reactive power compensation device itself to produce a certain amount of harmonic current, which SVC system filter design brings many difficulties. And SVG can be used in the bridge AC circuit multiplexing technology, multi-level technology or PWM technology to deal with to eliminate the lower number of harmonics, and the higher number of times such as 7,11 and other sub-harmonic reduction Small to acceptable level[3]. SVG dynamic reactive power compensation device not only produces very low harmonic content, but also to the load of harmonic and reactive power compensation.

3) Equipment loss

SVC dynamic reactive power compensation device itself, the topology and high-voltage inverter structure is similar, so the loss is basically the same. Voltage source inverter losses are generally small, SVG by a number of inverters in series, or in parallel composition, the total loss of the inverter part of the general does not exceed $0.8 \%$ of the total capacity. As the SVG capacity is only MCR, TCR half, FC loss only MCR, TCR half.

4) Adjust the characteristics

SVG can work in the rated tolerance to the rated capacity, so it is much wider than the SVC's operating range.

5) Area

Since there is no need for large capacity capacitors and reactors for energy storage components, SVG greatly reduces the size and footprint of the device. Under the same compensation capacity, SVG covers an area less than $1 / 2 \sim 2 / 3$ of SVC.

\section{Comparing results.}

SVG dynamic reactive power compensation device in all aspects are better than MCR-type SVC equipment and TCR-type SVC equipment.

\section{Application of Dynamic Reactive Power Compensation Device in Substation.}

In recent years, dynamic reactive power compensation device has been applied. SVC devices are widely used in wind power semi-outdoor substation, but SVG devices also gradually adopted among new user-substation in recent years [4]. The operation shows that the reactive power compensation device can adapt to the change of the system operation mode, so that the system voltage can be maintained at a reasonable high level in various ways, and improve the operation quality of the power grid. 


\section{Conclusion}

SVC dynamic reactive power compensation device, the current application of a wide range of reliable technology, economic and reasonable. But SVC also has its own weakness, it is impedance compensation, with the voltage drop its reactive power output will be reduced with the square of the voltage, and the loss of large, more harmonic. At present, the use of advanced intelligent control method to get the best compensation performance, is the world's advanced industrial countries and domestic scientific research institutions generally attach importance to the subject. SVG dynamic reactive power compensation device due to the fast response, low harmonic content, reactive power adjustment and other advantages, can greatly improve the power quality of the power grid, has now become the development of reactive power compensation technology. Although the SVG control method and the control system are more complex than the SVC, and because the larger number of larger capacity full control components, the price is higher than the use of ordinary thyristor SVC slightly higher, but with the GTO, IGCT, IGBT and other large SVG technology will gradually enter the mature application stage[5], as the latest generation of dynamic reactive power compensation technology, its continuous improvement of power quality of the power grid will play more and more significant The role of its metallurgical chemicals, iron, wind power and other industries have an immeasurable application prospects.

\section{References}

[1] Static dynamic reactive power compensation device (SVC) [J]. Jiang Feng. Science and Technology Innovation Guide. 2011 (20)

[2] Application of Static Dynamic Reactive Power Compensation Device (SVC) in Wind Farm [J]. Xu Guangrui, Chen Wang. Inner Mongolia Petrochemical Industry. 2011 (01)

[3] Design of a Novel Reactive Power Compensation Device [J]. Wang Fugong. Automation Technology \& Application. 2013 (07)

[4] Research and Design of Reactive Power Compensation Device [J]. Li Xiao, Bao Dudu, Duan Miao, Wei Ning, Qi Wenyan, Hu Yuanqi. Digital Technology and Application. 2013 (07)

[5] Dynamic Reactive Power Compensation Device Based on Magnetically Controlled Reactor [J]. Hubei Baoan, Jin Lili, Luo Yaqiao, Xu Bin, Fei Yijun, Yuan Xiaodong. Electric Power Automation Equipment 2010 (04) 